\title{
A REMARK ON COMPANIONSHIP AND PROPERTY P
}

\author{
MARTIN SCHARLEMANN
}

\begin{abstract}
If some nontrivial Dehn surgery on $\gamma$ yields a homotopy 3-sphere and $\kappa$ is a companion of $\gamma$, then some nontrivial Dehn surgery on $\kappa$ yields a homotopy 3-sphere.
\end{abstract}

DEFINITION. A knot $\kappa$ is a companion of a knot $\gamma$ in $S^{3}$ if $\gamma$ lies in a tubular neighborhood $\mathfrak{N}(\kappa)$ of $\kappa$, but not in any 3-cell contained in $\mathfrak{N}(\kappa)$. A knot $\gamma$ is simple if its only companions are itself and the unknot.

In [Go] it is shown that if some nontrivial Dehn surgery on a nontrivial knot yields a homotopy 3-sphere, then some nontrivial Dehn surgery on a nontrivial simple knot yields a homotopy 3-sphere. A more natural stronger statement is the following:

THEOREM 1. If some nontrivial Dehn surgery on $\gamma$ yields a homotopy 3-sphere and $\kappa$ is a companion of $\gamma$, then some nontrivial Dehn surgery on $\kappa$ yields a homotopy 3-sphere.

This stronger theorem was not yet supported by the technology of the time. The purpose of this note is to observe that it now follows immediately from the following recent theorem of Gabai:

THEOREM $2[\mathbf{G a}, 2.5]$. Let $(M, \partial M)$ be a Haken manifold, with $\partial M$ a union of tori, $(S, \partial S) \subset(M, \partial M)$ a Thurston norm-minimizing surface representing an element of $\mathrm{H}_{2}(M, \partial M)$, and $P$ a torus component of $\partial M$ disjoint from $S$. Then there is at most one way of attaching $D^{2} \times S^{1}$ to $P$ so that $S$ is not norm-minimizing in the resulting 3-manifold.

PROOF OF THEOREM 1 . If $\kappa$ is the unknot then any $1 / n$ surgery yields $S^{3}$, so henceforth assume $\kappa$ is nontrivial. Let $H$ and $N$ denote respectively the manifolds obtained from $S^{3}$ and $\mathfrak{N}(\kappa)$ by nontrivial Dehn surgery on $\gamma$. If $H$ is a homotopy 3 -sphere, then $\partial \mathfrak{N}(\kappa)$ must be compressible in $H$. Since it is incompressible in $H-N=S^{3}-\mathfrak{N}(\kappa)$, there is a compressing disk $\left(D^{2}, \partial D^{2}\right) \subset(N, \partial N)$.

Case 1. $\gamma$ is homologically nontrivial in $\mathfrak{N}(\kappa)$. Since $\gamma$ is homologically nontrivial in $\mathfrak{N}(k), \partial D^{2}$ is not a meridian of $\partial \mathfrak{N}(\kappa)$. Hence $H$ contains a direct summand which is obtained from $S^{3}$ by nontrivial Dehn surgery on $\kappa$.

Case 2. $\gamma$ is homologically trivial in $\mathfrak{N}(\kappa)$. Then a meridian of $\partial \mathfrak{N}(\kappa)$ is nullhomologous in $\mathfrak{N}(\kappa)-\mathfrak{N}(\gamma)$. Let $(S, \partial S) \subset(\mathfrak{N}(\kappa)-\mathfrak{N}(\gamma), \partial \mathfrak{N}(\kappa))$ be a surface of minimal Thurston norm representing the null-homology. Since $\kappa$ is a companion of $\gamma, S$ is not a disk, so it has nontrivial norm. By Theorem 2, the only Dehn filling

Received by the editors October 8, 1985.

1980 Mathematics Subject Classification. Primary 57M25.

The author was supported in part by a grant from the National Science Foundation. 
of $\partial \mathfrak{N}(\gamma)$ which reduces the norm is that which simply puts $\gamma$ in again. Hence for no other filling does $N$ contain a compressing disk.

REMARK. Gabai has anounced (December 1985) that the subtle combinatorics of $[\mathrm{Ga}, \S 3-7]$ applied to the $\partial$-reducing disk for $N$ shows also that in Case $1 \gamma$ is in fact the core. Thus satellite knots have homotopy property $\mathrm{P}$.

\section{REFERENCES}

[Ga] D. Gabai, Foliations and the topology of 3-manifolds. II, M.S.R.I. preprint 08912-85.

[Go] C. McA. Gordon, Dehn surgery and satellite knots, Trans. Amer. Math. Soc. 275 (1983), 687-708.

Department of Mathematics, UNiVersity of CALIFornia, SANTA Barbara, CALIFORNIA 93106 